\title{
In Vivo Tibial Fit and Rotational Analysis of a Customized, Patient-Specific TKA versus Off-the-Shelf TKA
}

\author{
Lennart Schroeder, CandMed ${ }^{1}$ Gregory Martin, MD² \\ ${ }^{1}$ Medizinische Fakultaet, Julius-Maximilians-Universitaet Wuerzburg, \\ Address for correspondence Lennart Schroeder, CandMed, \\ Wuerzburg, Germany \\ 2 Department of Orthopedic Surgery, Preferred Orthopaedics of the \\ Medizinische Fakultaet, Julius-Maximilians-Universitaet Wuerzburg, \\ Josef-Schneider-Straße 2, Wuerzburg 97080, Germany \\ Palm Beaches, Palm Beaches, Florida \\ (e-mail: lennartschroeder@gmx.de).
}

J Knee Surg 2019;32:499-505.

\begin{abstract}
Keywords

- customized implants

- implant fit

- implant rotation

- total knee arthroplasty

In total knee arthroplasty (TKA), surgeons often face the decision of maximizing tibial component fit and achieving correct rotational alignment at the same time. Customized implants (CIMs) address this difficulty by aiming to replicate the anatomical joint structure, utilizing data from patient-specific knee geometry during the manufacturing. We intraoperatively compared component fit in four tibial zones of a CIM to that of three different off-the-shelf (OTS) TKA designs in 44 knees. Additionally, we assessed the rotational alignment of the tibia using computed tomography (CT)-based computer aided design model analysis. Overall the CIM device showed significantly better component fit than the OTS TKAs. While $18 \%$ of OTS designs presented an implant overhang of $3 \mathrm{~mm}$ or more, none of the CIM components did $(p<0.05)$. There was a larger percentage of CIMs seen with optimal fit ( $\leq 1 \mathrm{~mm}$ implant overhang to $\leq 1 \mathrm{~mm}$ tibial bone undercoverage) than in OTS TKAs. Also, OTS implants showed significantly more component underhang of $\geq 3 \mathrm{~mm}$ than the CIM design (37 vs. 18\%). The rotational analysis revealed that $45 \%$ of the OTS tibial components showed a rotational deviation of more than 5 degrees and $4 \%$ of more than 10 degrees to a tibial rotational axis described by Cobb et al. No deviation was seen for the CIM, as the device is designed along this axis. Using the medial one-third of the tibial tubercle as the rotational landmark, $95 \%$ of the OTS trays demonstrated a rotational deviation of more than 5 degrees and $73 \%$ of more than 10 degrees compared with $73 \%$ of CIM tibial trays with more than 5 degrees and $27 \%$ with more than 10 degrees. Based on our findings, we believe that the CIM TKA provides both better rotational alignment and tibial fit without causing overhang of the tibial tray than the three examined OTS implants.
\end{abstract}

Numerous studies have demonstrated that both malalignment and improper fit of implant components during total knee arthroplasty (TKA) have significant impact on patients and are causative factors for pain and premature aseptic loosening leading to prosthesis failure and early revision. ${ }^{1-7}$ Mahoney and Kinsey reported that component overhang in TKA of $\geq 3 \mathrm{~mm}$ doubles the odds of clinically important

received

January 2, 2018

accepted

April 8, 2018

published online

May 25, 2018 knee pain 2 years after TKA. ${ }^{3}$ Furthermore, it has been shown that internal rotational errors of TKA components, especially of the tibia, are a major cause of pain and cause functional deficit after TKA. ${ }^{7,8}$ Maximizing fit and coverage of exposed tibial bone often results in implant malrotation. ${ }^{9}$ As such surgeons tend to downsize tibial components to address the obstacle of achieving correct tibial rotation without creating
Copyright $\odot 2019$ by Thieme Medical Publishers, Inc., 333 Seventh Avenue, New York, NY 10001, USA. Tel: +1(212) 584-4662.
License terms

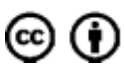

10.1055/s-0038-1653966. ISSN $1538-8506$ 
implant overhang. This however results in undercoverage of the cut tibial surface which has been theorized to contribute to component loosening, subsidence, and increased osteolysis from wear debris. ${ }^{10,11}$ Therefore, surgeons are often left with a compromise of having to choose between achieving optimal fit and proper tibial rotation.

There is an ongoing dispute whether asymmetric tibial implants provide better component fit and rotation than symmetric implants, and therefore, cause less pain with a better functional outcome after TKA. Martin et al stated that asymmetric components had fewer cases of internal malrotation as well as lesser magnitude of malrotation, thus performed better when maximizing tibial coverage. ${ }^{9}$ A study by Wernecke et al compared tibial bone coverage and percentage of overhang of the tibial tray of five symmetric and one asymmetric TKA designs. They reported that the asymmetric tray provided improved tibial coverage at the expense of posterolateral and posteromedial overhang of the tibial tray. ${ }^{12}$ Jin et al $^{13}$ showed improved surface coverage at the tibial medial-posterior dimension of an asymmetrical tibia due to its shape compared with standard symmetrical designs, whereas Incavo et $\mathrm{al}^{14}$ reported improved tibial coverage with symmetric designs.

Customized implants (CIMs) have been introduced as an option for patients undergoing TKA. Manufactured utilizing computed tomography (CT)-based imaging to provide patient-specific geometry, and these implants attempt to overcome the challenge of combining optimal bone coverage with correct rotational alignment of the implant.

The objective of this study was twofold: first to intraoperatively compare tibial fit of a CIM TKA implant (ConforMIS iTotal CR) to that of three different off-the-shelf (OTS) TKA designs; second to evaluate component rotation using CTbased imaging converted into computer aided design (CAD) models. We hypothesized that CIMs can provide better implant coverage of the tibial tray while maintaining proper rotational alignment.

\section{Materials and Methods}

\section{Intraoperative Measurements}

Forty-four knees (27 left and 17 right) of 44 patients were included in this single-center study. Of these, 26 were from male and 18 were from female. The mean age at surgery was 70.48 years with a range from 57 to 87 years. The average body mass index was 30.7 ranging from 22.0 to 49.1 . All patients underwent CT preoperatively for the CIM manufacturing process. The surgeries were performed by a single surgeon using the standard technique and indications for the CIM. After tibial preparation, a series of tibial trials from three OTS cruciate retaining TKA designs (Biomet Vanguard [OTS 1], Zimmer NexGen [OTS 2], and DePuy Sigma [OTS 3]) were placed on the cut tibial bone and aligned to the lateral tibial cortex, while trying to maintain proper rotational alignment and best component fit (-Fig. 1).

When trialing the tibia, the medial one-third of the tibial tubercle was used as an anatomical landmark for rotational purposes. Once rotationally aligned, if tibial underhang

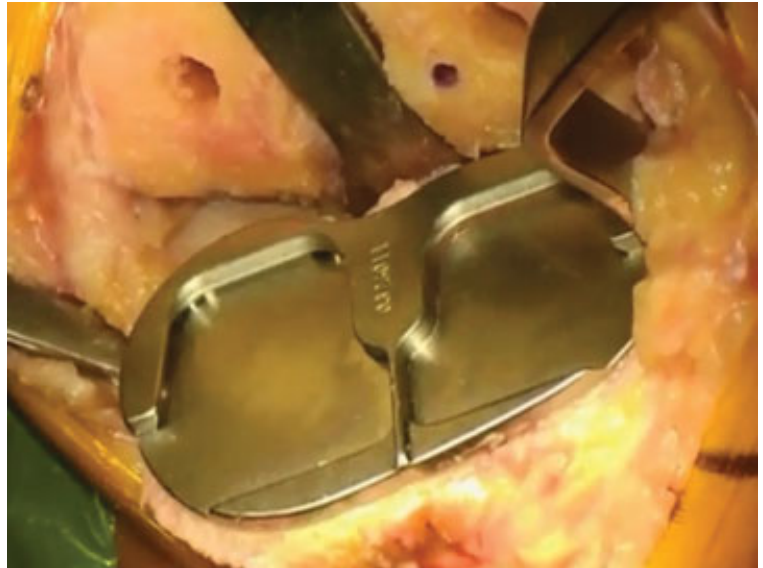

Fig. 1 Representative photograph of a placed tibial template aligned with the lateral tibial cortex. Tibial coverage was then measured in four different zones.

occurred, the component was upsized and if tibial overhang occurred, the tibial component was downsized.

Once the best-matched trial of each OTS TKA was obtained while maintaining proper rotation, the implant fit (overhang/underhang) was recorded in four tibial zones (medial-anterior, medial-posterior, lateral-anterior, and lateral-posterior). After all measurements were completed, the CIM tibial tray was implanted and measurements were repeated (-Fig. $\mathbf{2}$ ).

Instances of overhang were subdivided into absolute overhang ( $\geq 3 \mathrm{~mm}$ ) and relative overhang (1-2 $\mathrm{mm})$ and instances of underhang into absolute underhang $(>3 \mathrm{~mm}$ tibial bone undercoverage) and relative underhang (1-3 $\mathrm{mm}$ tibial bone undercoverage). An overhang of $\leq 1 \mathrm{~mm}$ and undercoverage of $\leq 1 \mathrm{~mm}$ were considered to be optimal fit.

\section{CAD Analysis}

Preoperatively, CT-based imaging of the knee was converted into a three-dimensional CAD model to provide the patientspecific geometry for the manufacturing process of the CIMs. Utilizing these models, simulated surgery was performed.

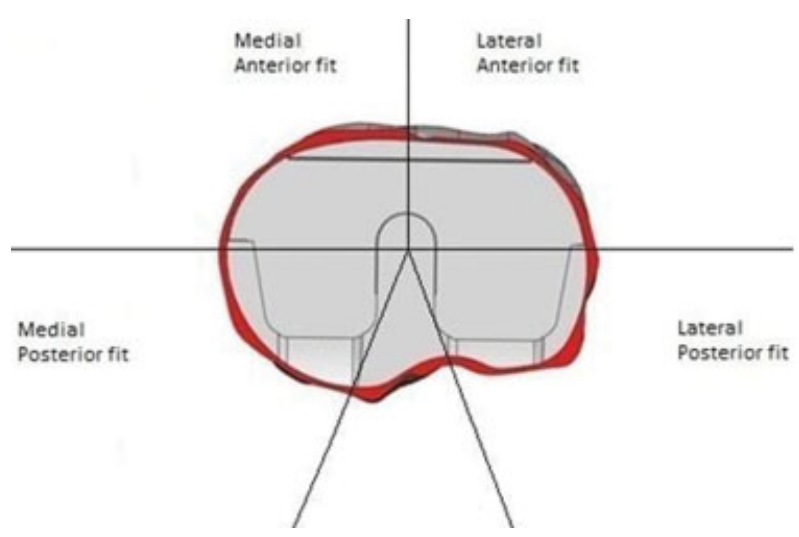

Fig. 2 Tibial bone undercoverage and implant overhang was measured in the four depicted zones. 

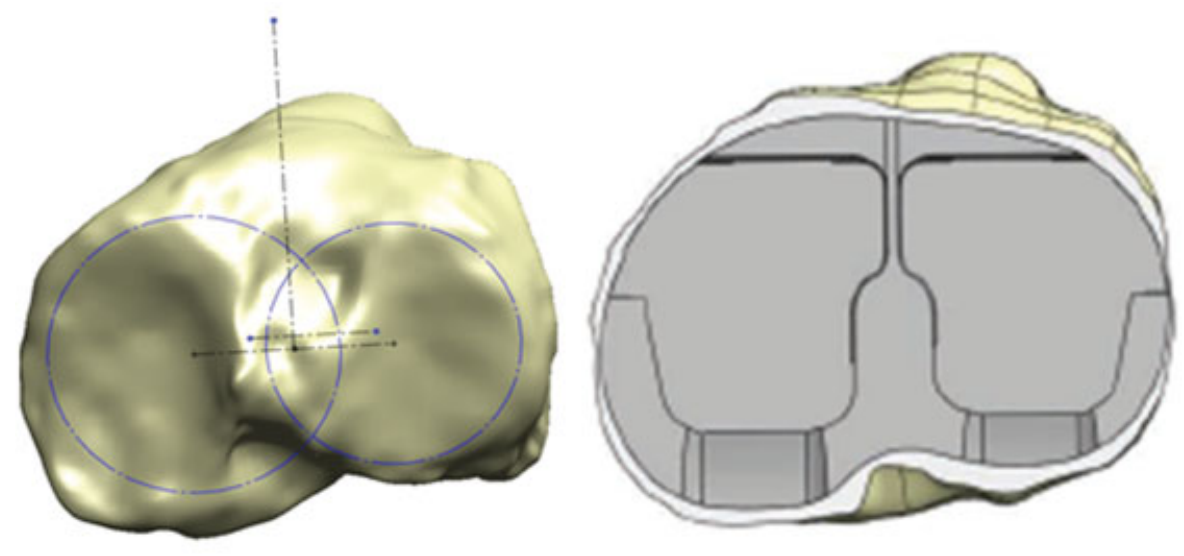

Fig. 3 Patient-specific CAD image.

Tibial trays for all OTS implants were set for maximal bone coverage while ignoring rotational errors. The optimal tibial trial was chosen based on providing maximum bone coverage without overhang. Once the optimal size was determined, rotational deviation of implant alignment, if any, was computed utilizing two different alignment methods. The first method was to align to an axis described by Cobb et $\mathrm{al}^{15}$ defined as the perpendicular to the line connecting the medial and lateral tibial plateau centers ( - Fig. 3 ) and the second to an axis defined as the line connecting the center of the tibial plateau and the medial one-third of the tibial tubercle. The degrees of variance from these lines were then measured (-Fig. 4).

To determine the significance between data in each group, a statistical analysis was performed either by using inbuilt or custom functions in Microsoft Excel 2016 (Microsoft Corp, Redmond, WA).

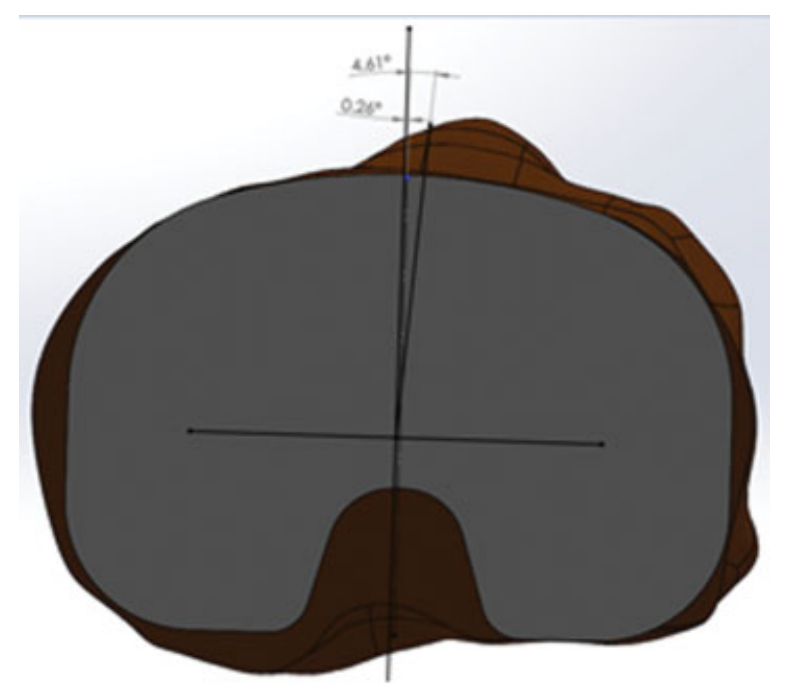

Fig. 4 Representation of the CAD analysis depicting the difference in tibial rotation between the tibial 3rd and Cobb landmarks.

\section{Results}

\section{Intraoperative Fit Analysis}

The tibial fit analysis revealed that $37 \%$ of the OTS implants showed tibial undercoverage of more than $3 \mathrm{~mm}$ in at least one of the examined tibial zones. There was no significant difference between the OTS implant types: OTS 1 implant showed absolute underhang in 36\%, OTS 2 in 34\%, and OTS 3 in $41 \%$ of the trial cases. The CIM tibial component showed absolute underhang in $18 \%$ of the knees which was significantly less in comparison to the OTS tibial components $(p=0.025)$. In the three OTS groups, tibial undercoverage was most frequently seen in the medial-posterior zone while the distribution of undercoverage for the CIM was seen equally frequent in the medial-posterior and medial-anterior zone of the tibial bone (-Fig. 5).

In spite of the surgeon's preference for accepting tibial undercoverage rather than tibial component overhang, significant overhang of $\geq 3 \mathrm{~mm}$ of the tibial component in any zone was seen in $16 \%$ of the OTS $1,20 \%$ of the OTS 2 , and $18 \%$ of the OTS 3 TKAs. None $(0 \%)$ of the CIM TKA trials experienced tibial tray overhang of $\geq 3 \mathrm{~mm}$, which was found to be statistically significant $(p<0.001)(-$ Fig. 6$)$.

For the four zones analyzed, optimal fit $(\leq 1 \mathrm{~mm}$ implant overhang to $\leq 1 \mathrm{~mm}$ tibial bone undercoverage) and relative underhang (1-3 mm tibial bone undercoverage) of the tibial component were seen in $80 \%$ of CIM TKAs and $27 \%$ of TKAs in the three OTS groups on average $(23,25$, and $34 \%$ for OTS 1 , OTS 2 , and OTS 3 implants, respectively). These differences were found to be statistically significant, with a larger percentage of patients experiencing optimal fit or relative underhang with the CIM TKA trials when compared with each of the three OTS groups $(p<0.001)$ ( - Fig. 7 ).

Setting the threshold for overhang to more than $1 \mathrm{~mm}$ showed that an average of $35 \%$ of the three OTS and $2 \%$ of the CIM tibial trays exhibited tibial component overhang. This difference was shown to be of statistical significance $(p<0.0001)$. Within the three OTS implant types, the OTS 3 tibial tray was found to have overhang of more than $1 \mathrm{~mm}$ less often (25\%) than both the OTS 1 and OTS 2 TKA trays 


\section{Underhang $>3 \mathrm{~mm}$}
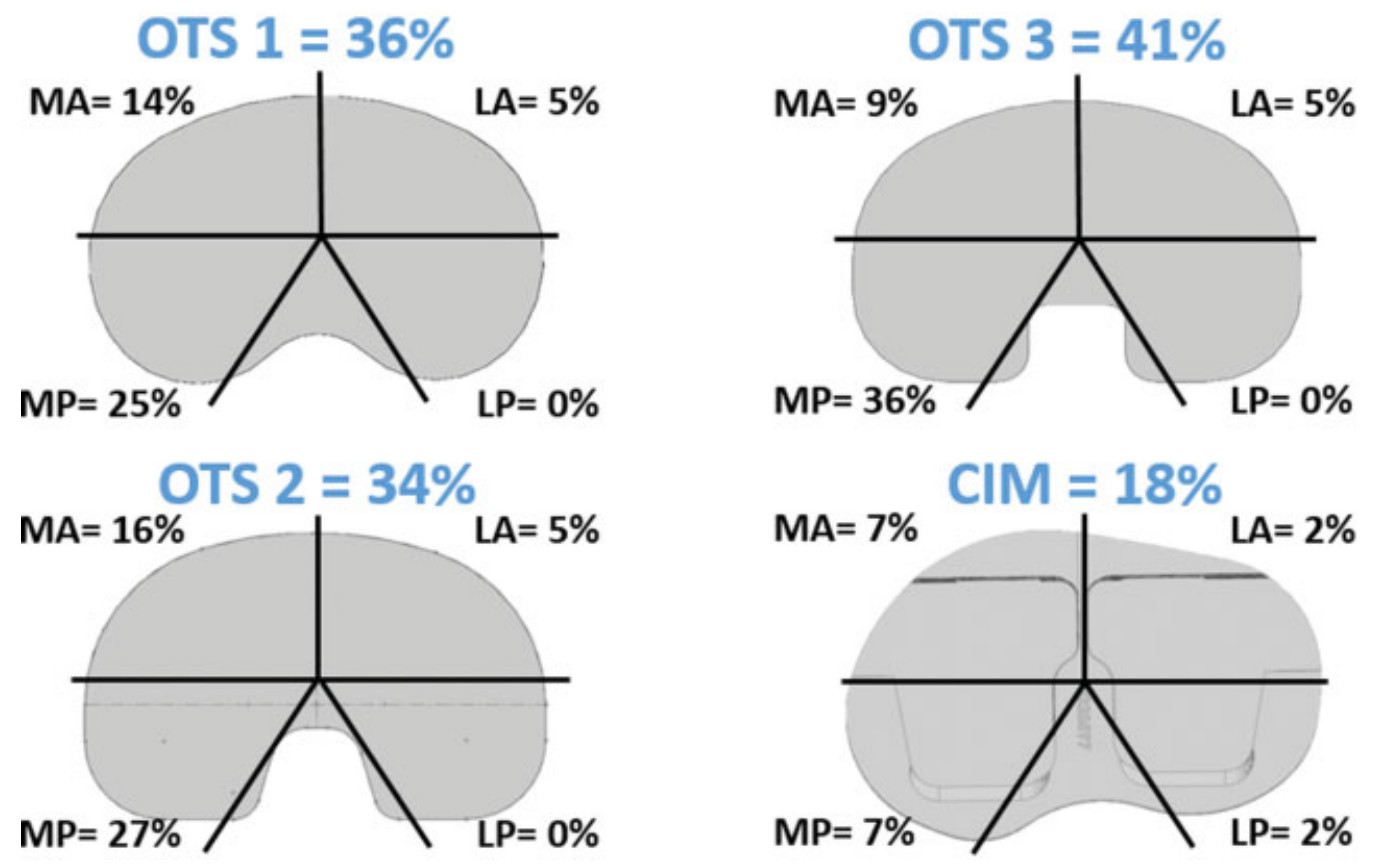

Fig. 5 Tibial undercoverage observed in the four depicted areas. Underhang was most frequently seen in the medial-posterior zone.

\section{Overhang $\geq 3 \mathrm{~mm}$}
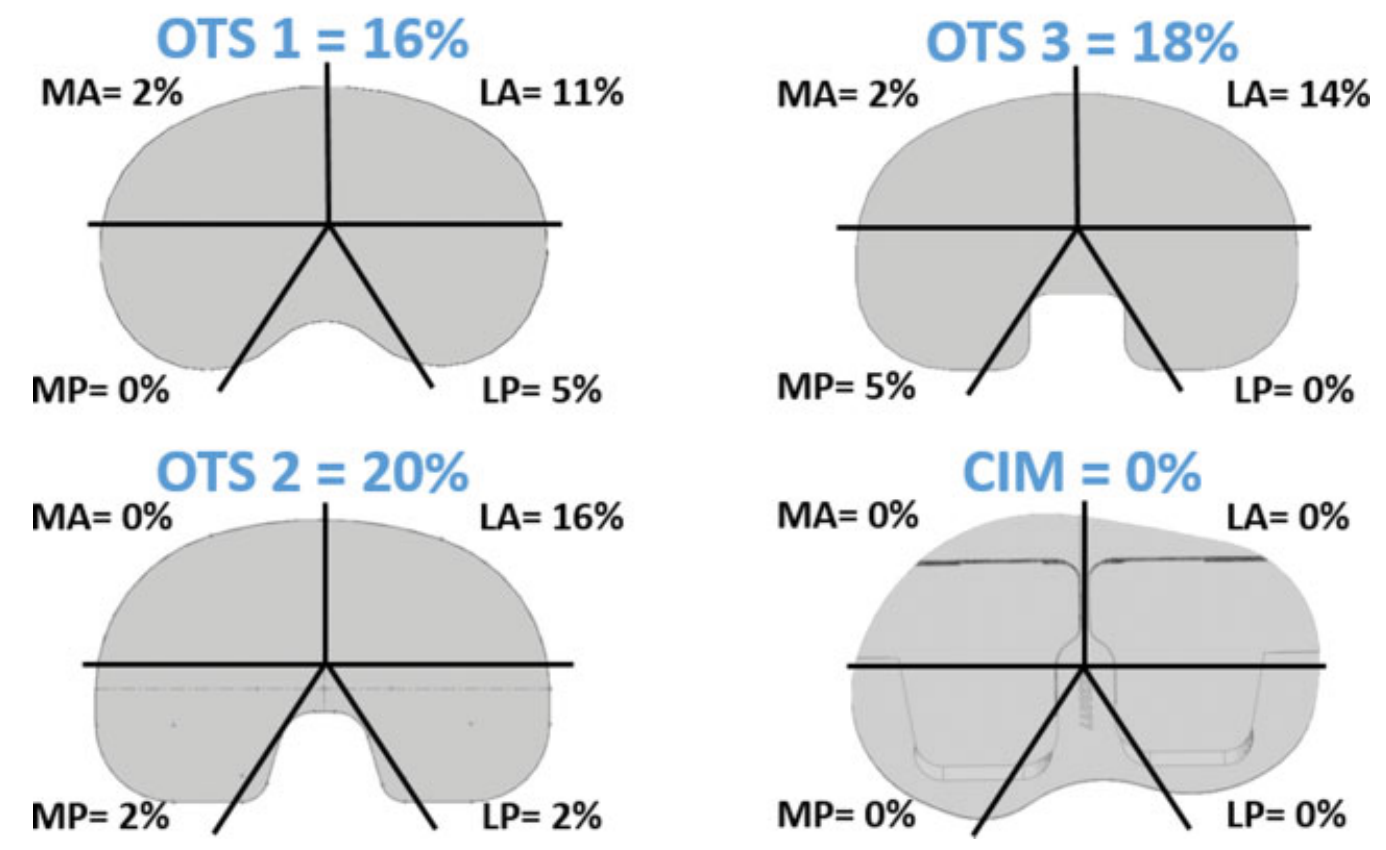

Fig. 6 Implant overhang observed in the four depicted areas. Overhang was most frequently seen in the lateral-anterior zone.

(both 42\%). However, these were not found to be statistically significant ( $p=0.173)$.

Finally, there were individual cases among the OTS groups where significant overhang and underhang were seen for the same tibial trial or underhang was evident in more than one zone. There were no such cases with the CIM tibial trays (-Figs. 5 and 6).

\section{Rotation}

When the tibial trays were placed for maximal tibial coverage using the CT-based simulation of the preoperatively designed CAD models, data revealed that the average rotational deviation of the OTS TKA trays to a tibial axis described by Cobb et al was 4.92 degrees of internal rotation (range: 0.08-12.06 degrees) with $45 \%$ of the tibial components 


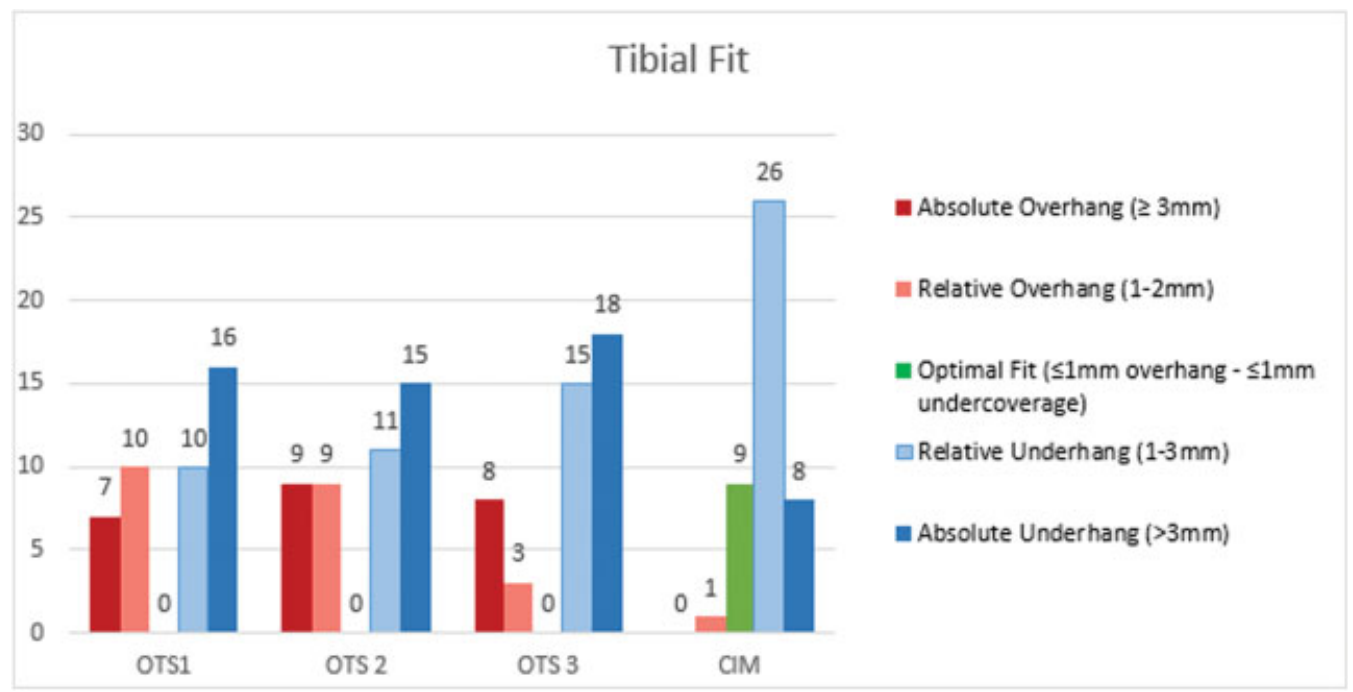

Fig. 7 The results of the tibial fit analysis are displayed in this figure.

having a rotational deviation of more than 5 degrees $(p<0.001)$ and $4 \%$ with a rotational deviation of more than 10 degrees internal rotation. As the CIM is designed to align along the axis described by Cobb et al, no rotational deviation ( 0 degree) was seen in the CAD simulation. When utilizing the medial one-third of the tibial tubercle as the rotational landmark, the average rotational deviation of the OTS tibial trays was seen to be much greater, with an average of 12.62 degrees of internal rotation (range: 2.1220.21 degrees) with $96 \%$ of the tibial components having a rotational deviation of more than 5 degrees and $73 \%$ with a rotational deviation of more than 10 degrees internal rotation. For the CIM, a rotational deviation to the medial onethird of the tibial tubercle based on the CAD analysis was recorded with an average of 7.69 degrees of internal rotation (range: 0.97-15.34 degrees). The difference in the average rotational error between the OTS and CIM arms was found to be statistically significant $(p<0.001) ; 75 \%$ patients with the CIM had a rotational deviation of more than 5 degrees and $27 \%$ of more than 10 degrees internal rotation ( - Table $\mathbf{1})$.

When assessing the results of the CAD model sizing changes of the tibial components to achieve best possible component fit for the OTS tibial trays, we found that $47 \%$ had to be either up- or downsized as compared with those actually trialed in surgery. Furthermore, among the three different OTS TKA trays, $16 \%$ of the OTS 1 tibial trays had to be changed in size, and $25 \%$ of the OTS 2 and all (100\%) OTS 3 tibial trays were either up- or downsized to achieve the best component fit. The percentage of changes in the OTS 1 and OTS 2 groups was significantly lower than the number of changes required in the OTS 3 group $(p<0.001)$.

\section{Discussion}

Improper fit and malrotation of the tibial component during TKA have been shown to cause patellofemoral complications, implant loosening, rotational instability, and pain leading to early revisions. ${ }^{6,7,10,16,17}$ Although TKA is a well-established procedure in orthopaedic surgery with approximately 700.000 knee replacements done annually alone in the United States, ${ }^{18}$ around $20 \%$ of patients report dissatisfaction with their TKA result due to multiple reasons such as pain, unfulfilled patient expectations, and functional impairment. ${ }^{19-23}$

To minimize these problems, the CIM has been designed to improve tibial implant coverage while maintaining the

Table 1 Results of the implant rotational analysis

\begin{tabular}{|l|l|l|l|l|}
\hline \multicolumn{2}{|c|}{} & CIM (deg) & OTS (deg) & $p$-Value \\
\hline \multirow{4}{*}{ Cobb et al $^{15}$} & Average & 0 & 4.92 & $<0.001$ \\
\cline { 2 - 5 } & $\%>5 \mathrm{deg}$ & 0 & 45 & $<0.001$ \\
\cline { 2 - 5 } & $\%>10 \mathrm{deg}$ & 0 & 4 & 0.333 \\
\hline \multirow{3}{*}{ Medial one-third } & Average & 7.69 & 12.62 & $<0.001$ \\
\cline { 2 - 5 } & $\%>5 \mathrm{deg}$ & 75 & 96 & $<0.001$ \\
\cline { 2 - 5 } & $\%>10 \mathrm{deg}$ & 27 & 73 & $<0.001$ \\
\hline
\end{tabular}

Abbreviations: CIM, customized implant; OTS, off-the-shelf. 
rotational alignment of the tibial tray proposed by Cobb et al by using patient-specific anatomical markers in the design process. We believe this is the first study to look at both the difference of tibial component rotation and fit of the tibial tray of a CIM, TKA implant design compared with three different OTS TKAs.

This study has several limitations. First, all TKAs and intraoperative measurements were done by a single surgeon which may affect the results when measuring tibial bone coverage of the three OTS implants from a surgical technique standpoint. However, the surgeon had used all of these implants previously and was especially experienced with the OTS 3 and CIM brands.

A second limitation is that patient-specific jigs manufactured for the iTotal CR were used for the tibial bone resection. Yet, this tibial cut is similar to any other cut in the resulting shape of the cut tibial bone. Third, as the CAD analysis of the rotational deviation from an axis described by Cobb et $\mathrm{al}^{15}$ and an axis to the medial one-third of the tibial tubercle was performed manually and for each implant individually. This may have resulted in intraobserver mistakes. Varying opinions exist on what landmarks to use when assessing component alignment. We did not utilize all methods of tibial component rotation, only methods based on the location of the tibial tubercle and by an axis described by Cobb et al which we believe is accurate from what multiple studies have reported. ${ }^{1,15}$ Aligning the OTS tibial components toward the medial one-third of the tubercle has been shown in multiple studies to be the most reproducible clinical landmark in terms of tibial tray rotation and is used by the majority of surgeons in the United States for tibial alignment. ${ }^{6,24}$ We only evaluated three OTS implant designs for this study although there are many more different types on the market. Nonetheless, based on our results and similarities between the OTS brands, we feel these results are likely highly translatable to other OTS brands. In this study, only symmetrical implant designs were compared with the CIM TKA, despite the fact that implant manufacturers have introduced other asymmetrical designs on the market. However, as Jin et al emphasized in his study, although leading to better results in tibial fit, there were still cases with both over- and underhang on the same tibial trial with the asymmetrical design. $^{13}$

It has to be stated that no precise definitions for absolute tibial component underhang or overhang can be found in the literature. However, Mahoney and Kinsey's observations indicate that the presence of an overhang of $\geq 3 \mathrm{~mm}$ in at least one zone increases the odds of patients reporting knee pain which is why we chose this threshold to be of importance. ${ }^{3}$ Jin et al $^{13}$ suggested underhang is more acceptable during surgery than overhang as the surgeon can remove uncovered bone during the procedure and correct rotation. To our knowledge, no studies investigating a possible correlation between tibial undercoverage and implant failure exist. However, it has been hypothesized that tibial undercoverage may be a causal factor in increased osteolysis, tibial subsidence and implant loosening, and therefore lead to pain and early implant failure. ${ }^{10,11,25}$ Additionally, studies have shown that blood exudation from exposed bone sections not covered by prostheses are an important source of blood loss and that the control of bleeding is not amenable to methods such as electrocautery, ligature control, or the use of bone wax. ${ }^{26-28}$ We suggest that further research should be made in this field.

Our study supports Jin et al's and Wernecke et al's findings that asymmetric tibial designs improve surface coverage of the cut tibial bone compared with symmetric implants. ${ }^{12,13}$ Due to the naturally larger medial plateau compared with the lateral tibial plateau, a difference in coverage between the CIM and the three OTS implants was particularly striking to be seen in the medial-posterior zone. This matches Jin's findings when comparing an anatomical tibial design with three symmetrical designs. The CIM design showed no overhang of $\geq 3 \mathrm{~mm}$ in any of the cases compared with $18 \%$ of the OTS implants and additionally provided better tibial coverage, meaning less undercoverage than the OTS trays in any of the four observed zones. However, this contrasts Wernecke et al's ${ }^{12}$ observations of asymmetric trays only providing the highest tibial coverage at the expense of having posterolateral and posteromedial overhang of the tibial tray. This further reinforces that a fixed tibial asymmetry presents the same sizing and rotational challenges as do fixed symmetrical implants.

The data analysis revealed that the majority of the three OTS implants had to be undersized to prevent overhang of the cortical bone and to maintain proper rotational alignment. The CIM showed significantly less underhang of more than $3 \mathrm{~mm}$ which might be a factor in better long-term outcome; however, additional studies will be needed to confirm this.

When taking a closer look at patients who experienced either minor tibial undercoverage $(1-3 \mathrm{~mm})$ or what we defined as "optimal" implant fit $(\leq 1 \mathrm{~mm}$ overhang to $\leq 1 \mathrm{~mm}$ tibial underhang), we discovered that $59 \%$ of the CIM TKAs showed tibial bone undercoverage of 1 to $3 \mathrm{~mm}$ in one of the four measured tibial zones. We believe that these findings are caused by the fact that the CIM is designed to accommodate $\mathrm{a}+/ \mathrm{-}-5$ degrees of rotational flexibility from set rotation without causing overhang. This grants the surgeon flexibility for the tibial alignment but also results in minor undercoverage of the tibial bone in some cases.

Martin et al described in their study that the majority (70\%) of tibial components aligned toward the middle of the medial one-third of the tubercle (medial one-sixth) were internally malrotated by an average of $9 \pm 7$ degrees when coverage was maximized. ${ }^{9}$ With the three OTS tibial trays showing an average rotational error of 13 degrees to the medial one-third when maximized for coverage, our results are similar to these findings. According to Nicoll and Rowley, internal rotational errors of the tibial component were a major cause for pain and functional deficit after total knee replacement. ${ }^{7}$ Furthermore, previous work from Berger and Rubash reported that internal rotation of the tibial component from 3 to 8 degrees correlated with patellar subluxation, whereas internal rotation of 7 to 17 degrees correlated with findings of patellar dislocation or patellar prosthesis 
failure. ${ }^{6}$ Referring to their results, the majority of patients in our study cohort treated with an OTS tibial tray would have been at the risk, if tibial components were not downsized and externally rotated to prevent malalignment. When analyzing the data, we found several cases of $6-9 \mathrm{~mm}$ of tibial bone undercoverage with OTS tibial trays set for what the senior surgeon believed to be correct tibial rotation. A large undercovered surface intraoperatively may be a concerning event leading to the surgeon upsizing the tibial component. This could result in the tibial component being internally rotated to provide better coverage and may be the source of poorer outcomes as suggested by previous studies investigating the relationship between tibial rotation and patient outcomes. ${ }^{5,7}$ Being patient specifically designed to align to the Cobb et al's axis, the rotational deviation to the medial one-third of the CIM was dependent on the degree difference between both axes. Lawrie et al examined different previously described axes and came to the conclusion that no single anatomic axis or landmark guaranteed correct alignment of the tibial component. ${ }^{1}$ They reported correct alignment of the tibial component to be best achieved by splitting the difference between the medial third of the tibial tubercle and the medial-lateral axis of the resected tibial surface described by Cobb et al. ${ }^{15}$ As the CIM allows $+/-5$ degrees of rotational flexibility without causing overhang, we believe the CIM TKA has a better ability to achieve optimal tibial rotation while maintaining proper fit when compared with OTS implants investigated in this study.

\section{Conflict of Interest}

Mr. Schroeder's research and internship is funded by ConforMIS Inc. Dr. Martin reports personal fees from ConforMIS Inc., during the conduct of the study.

\section{References}

1 Lawrie CM, Noble PC, Ismaily SK, Stal D, Incavo SJ. The flexionextension axis of the knee and its relationship to the rotational orientation of the tibial plateau. J Arthroplasty 2011;26(6, Suppl):53-58

2 Howell SM, Chen J, Hull ML. Variability of the location of the tibial tubercle affects the rotational alignment of the tibial component in kinematically aligned total knee arthroplasty. Knee Surg Sports Traumatol Arthrosc 2013;21(10):2288-2295

3 Mahoney OM, Kinsey T. Overhang of the femoral component in total knee arthroplasty: risk factors and clinical consequences. J Bone Joint Surg Am 2010;92(05):1115-1121

4 Sharkey PF, Hozack WJ, Rothman RH, Shastri S, Jacoby SM. Insall Award paper. Why are total knee arthroplasties failing today? Clin Orthop Relat Res 2002;(404):7-13

5 Barrack RL, Schrader T, Bertot AJ, Wolfe MW, Myers L. Component rotation and anterior knee pain after total knee arthroplasty. Clin Orthop Relat Res 2001;(392):46-55

6 Berger RA, Rubash HE. Rotational instability and malrotation after total knee arthroplasty. Orthop Clin North Am 2001;32(04): 639-647

7 Nicoll D, Rowley DI. Internal rotational error of the tibial component is a major cause of pain after total knee replacement. J Bone Joint Surg Br 2010;92(09):1238-1244

8 Berger RA, Crossett LS, Jacobs JJ, Rubash HE. Malrotation causing patellofemoral complications after total knee arthroplasty. Clin Orthop Relat Res 1998;356(356):144-153
9 Martin S, Saurez A, Ismaily S, Ashfaq K, Noble P, Incavo SJ. Maximizing tibial coverage is detrimental to proper rotational alignment. Clin Orthop Relat Res 2014;472(01):121-125

10 Chau R, Gulati A, Pandit H, et al. Tibial component overhang following unicompartmental knee replacement-does it matter? Knee 2009;16(05):310-313

11 Hitt K, Shurman JR II, Greene K, et al. Anthropometric measurements of the human knee: correlation to the sizing of current knee arthroplasty systems. J Bone Joint Surg Am 2003;85-A(04, Suppl 4):115-122

12 Wernecke GC, Harris IA, Houang MT, Seeto BG, Chen DB, MacDessi SJ. Comparison of tibial bone coverage of 6 knee prostheses: a magnetic resonance imaging study with controlled rotation. J Orthop Surg (Hong Kong) 2012;20(02):143-147

13 Jin C, Song EK, Prakash J, Kim SK, Chan CK, Seon JK. How much does the anatomical tibial component improve the bony coverage in total knee arthroplasty? J Arthroplasty 2017;32(06):1829-1833

14 Incavo SJ, Ronchetti PJ, Howe JG, Tranowski JP. Tibial plateau coverage in total knee arthroplasty. Clin Orthop Relat Res 1994; (299):81-85

15 Cobb JP, Dixon H, Dandachli W, Iranpour F. The anatomical tibial axis: reliable rotational orientation in knee replacement. J Bone Joint Surg Br 2008;90(08):1032-1038

16 Lynch AF, Rorabeck CH, Bourne RB. Extensor mechanism complications following total knee arthroplasty. J Arthroplasty 1987 2(02):135-140

17 Rand JA. The patellofemoral joint in total knee arthroplasty. J Bone Joint Surg Am 1994;76(04):612-620

18 Kurtz S, Ong K, Lau E, Mowat F, Halpern M. Projections of primary and revision hip and knee arthroplasty in the United States from 2005 to 2030. J Bone Joint Surg Am 2007;89 (04):780-785

19 Noble PC, Conditt MA, Cook KF, Mathis KB. The John Insall Award: Patient expectations affect satisfaction with total knee arthroplasty. Clin Orthop Relat Res 2006;452(452):35-43

20 Bourne RB, Chesworth BM, Davis AM, Mahomed NN, Charron KD. Patient satisfaction after total knee arthroplasty: who is satisfied and who is not? Clin Orthop Relat Res 2010;468(01): 57-63

21 Iorio R, Robb WJ, Healy WL, et al. Orthopaedic surgeon workforce and volume assessment for total hip and knee replacement in the United States: preparing for an epidemic. J Bone Joint Surg Am 2008;90(07):1598-1605

22 Wright J, Ewald FC, Walker PS, Thomas WH, Poss R, Sledge CB. Total knee arthroplasty with the kinematic prosthesis. Results after five to nine years: a follow-up note. J Bone Joint Surg Am 1990;72(07):1003-1009

23 Ranawat CS. The patellofemoral joint in total condylar knee arthroplasty. Pros and cons based on five- to ten-year follow-up observations. Clin Orthop Relat Res 1986;(205):93-99

24 Coughlin KM, Incavo SJ, Churchill DL, Beynnon BD. Tibial axis and patellar position relative to the femoral epicondylar axis during squatting. J Arthroplasty 2003;18(08):1048-1055

25 Servien E, Saffarini M, Lustig S, Chomel S, Neyret P. Lateral versus medial tibial plateau: morphometric analysis and adaptability with current tibial component design. Knee Surg Sports Traumatol Arthrosc 2008;16(12):1141-1145

26 Demey G, Servien E, Pinaroli A, Lustig S, Ait Si Selmi T, Neyret P. The influence of femoral cementing on perioperative blood loss in total knee arthroplasty: a prospective randomized study. J Bone Joint Surg Am 2010;92(03):536-541

27 Kim HJ, Fraser MR, Kahn B, Lyman S, Figgie MP. The efficacy of a thrombin-based hemostatic agent in unilateral total knee arthroplasty: a randomized controlled trial. J Bone Joint Surg Am 2012; 94(13):1160-1165

28 Moo IH, Chen JYQ Pagkaliwaga EH, Tan SW, Poon KB. Bone wax is effective in reducing blood loss after total knee arthroplasty. J Arthroplasty 2017;32(05):1483-1487 